\title{
A mathematical model for the accumulation of fatigue in bars subjected to lateral vibrations
}

\author{
Petru Cardei ${ }^{1,}{ }^{*}$, Raluca Sfiru ${ }^{1}$, and Vergil Muraru ${ }^{1}$ \\ ${ }^{1}$ INMA Bucharest, Computerized Engineering Department, 013813 Bucharest, Romania
}

\begin{abstract}
The article presents a mathematical model for the phenomenon of fatigue accumulation in the slender bar subjected to lateral bending. The model is based on the Euler-Bernoulli type bar, a bi-linear elastic-plastic model and, for simulation of fatigue, a system of equations describing the decrease resistance parameters of the material: the ultimate strain and stress. In the article is exposes the bar response to two types of dynamic loads, as well as a fatigue test simulating process using the proposed model, which results in the Wöhler diagram of the material for the bending vibrations. The conclusions outline the outlook of the model as well as its shortcomings. The author expounds the advantages of the model, but the reader is also challenged to reflect on the opportunity of using mathematical models of great complexity.
\end{abstract}

\section{Introduction}

Mathematical modelling in the field of material fatigue occupies a central place in the literature dedicated to this problem. Starting from the elementary mathematical model of the $S$ - $N$ curve, also known as Wöhler curve, [1], up to contemporary mathematical models based on differential equations and partial and even more complicated derivatives, [2-5], [8-11], the purpose of mathematical modelling in this area was to predict the ceding of materials or to specify a period of time during which the material will not yield under certain conditions, or will yield with a very low probability.

The model presented in these article attempts, with simple means, to adapt to the problem of materials fatigue, mathematical models developed to simulate the memory of materials or mathematical models of materials that change their qualities during operation, [6-7]. The results presented in this article represent a stage of development towards models that consider two-dimensional and three-dimensional structures. It is also the application of the model in structural analysis programs that work through different numerical methods.

\section{Problem Formulation}

The subject of this article is to model the fatigue of bars subjected to transverse vibrations. For this reason a metallic bar of length, $L=0.3 \mathrm{~m}$, having the circular section with the radius $r=0.0025 \mathrm{~m}$ (see Figure 1) will be considered. The material from which the bar is built is

\footnotetext{
* Corresponding author: petru cardei@yahoo.com
} 
steel with the following characteristics: Young's modulus $E=2.1 \cdot 10^{11} \mathrm{~Pa}$, Poisson's ratio, $\nu=0.29$, and mass density, $\rho=7850 \mathrm{Kg} / \mathrm{m} 3$.

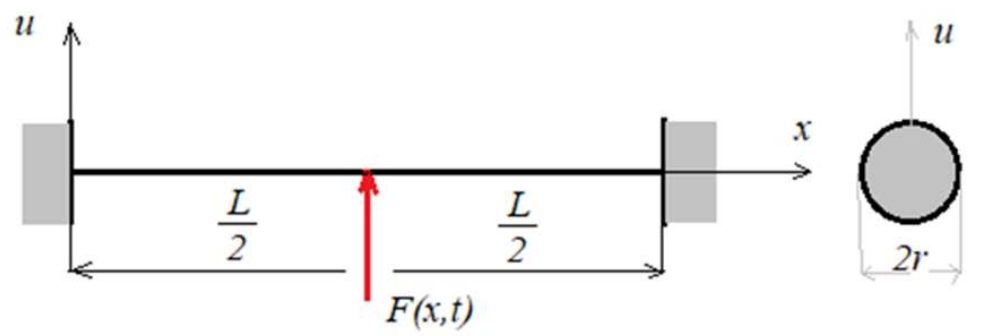

Fig. 1 Geometry, border condition and load of the model.

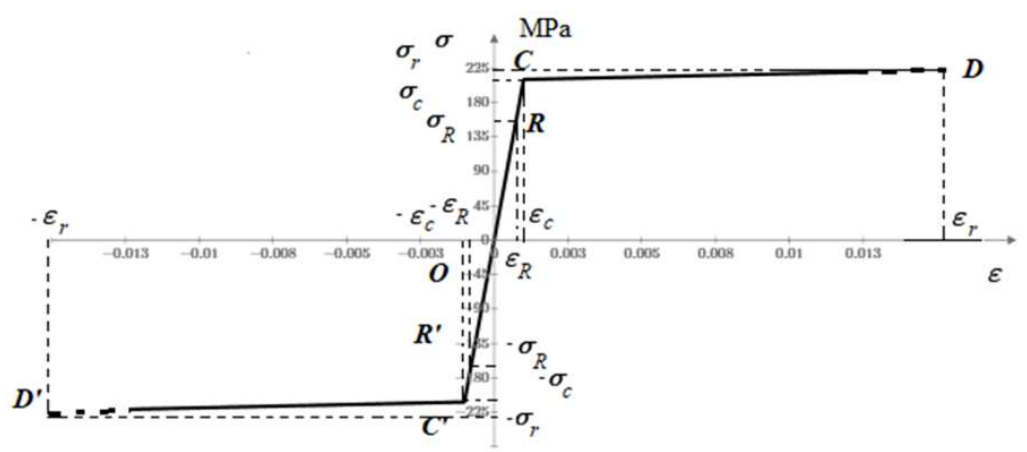

Fig. 2 The Stress-Strain curve of the material of the beam.

The material of the beam is modelled as a linear Strain-Hardening Stress-Strain material (bilinear curve, [8], see Figure 2) having for the tangent modulus, [9], the value $E_{p}=843.37$ $\mathrm{MPa}$, yield strain, $\varepsilon_{c}=0.001$, yield strength, $\sigma_{c}=210 \mathrm{MPa}$, ultimate tensile strain, $\varepsilon_{r}=0.25$, and by the ultimate tensile strength, $\sigma_{r}=420 \mathrm{MPa}$. Resistance to fatigue through any bending cycle of the material, [10], is characterized by the stress $\sigma_{R}=168 \mathrm{MPa}$, and by the strain $\varepsilon_{R}=0.0008$ (both are the fatigue limit, [11]).

\subsection{The equations of the bar deformation}

To solve the equations of the transverse vibrations of the bar in Figure 1, are used the model Euler-Bernoulli, [12] or [13], for a prismatic bar having the flexural rigidity , the cross section area, $A$, the second moment of area, $I$, mass density, $\rho$, all constant along the length of the bar:

$$
E I \frac{\partial^{4} y}{\partial x^{4}}=-\rho A \frac{\partial^{2} y}{\partial t^{2}}
$$

The bar is assumed built-in at both ends. Consequently, after [12], boundary conditions are:

$$
y(0, t)=0, y(L, t)=0, \partial y / \partial x(0, t)=0, \quad \partial y / \partial x(L, t)=0
$$

The initial conditions are: 


$$
y(x, 0)=0, \frac{\partial y}{\partial t}(x, 0)=0
$$

The force that produces the vibration of the bar is given by the formula:

$$
F(x, t)=F_{0} \sin \omega t H\left(x-\frac{L}{2}\right)
$$

where $H$ is the Heaviside function.

\subsection{Rheological equations}

The rheological behaviour of the bar material is defined by the equations (5) - (9), after [7].

$$
\begin{aligned}
& \sigma(\varepsilon)=\left\{\begin{array}{c}
E \varepsilon, \text { if }|\varepsilon|<\varepsilon_{c} \\
E_{p}\left(\varepsilon-\varepsilon_{c}\right)+\sigma_{c}, \text { if } \varepsilon_{c} \leq|\varepsilon| \leq \varepsilon_{r}
\end{array}\right. \\
& \varepsilon_{r}=\varepsilon_{r}^{0}-\frac{1}{E_{p}} \int_{0}^{t} w(x, s) d s \\
& \sigma_{r}=\sigma_{r}^{0}-\int_{0}^{t} w(x, s) d s \\
& E_{p}=\frac{\sigma_{r}-\sigma_{c}}{\varepsilon_{r}-\varepsilon_{c}} \\
& \left.w(x, t)=|| \sigma(x, t)\left|-\sigma_{R}\right| \cdot\left|\frac{\partial \varepsilon}{\partial t}(x, t)\right| \cdot H(\mid \varepsilon(x, t)) \mid-\varepsilon_{R}\right)
\end{aligned}
$$

It is easy to see that the relationships (5) - (9) imply that the tangent modulus, $E_{p}$ remains constant in the transformation suffered by the bar material in space and time. The mathematical model (5) - (9) for the fatigue of the material, takes into account the statement made in the literature, [10], that fatigue is defined as a sensitive reduction of the mechanical characteristics of the material. In the case of this model, it means lowering of the ultimate strength, both in strain and in stress.

\section{Problem Solution}

In this article, it is assumed that the loads applied to the bar, produce in its material, stresses and strain located in the elastic-linear area, reaching at most the point of flow (point $C$ on the characteristic curve of the material, shown in Figure 2). The hypothesis of the validity of the linear summation of the effects of several requests will also be accepted. With these specifications, the solution of the equation (1) with the boundary conditions (2), the initial conditions (3) and the loading (4), is obtained in agreement with [13]:

$$
y(x, t)=\sum_{i=1}^{N_{f}} \sum_{k=1}^{N_{c}} C_{i k} Y_{i}(x) \psi_{k}(t),
$$


where $N_{f}$ is the number of bar pulsation considered in the calculus (in this case 9 , indexed by $i), N_{c}$ is the number of sinusoidal components of the disturbing force (indexed with the index $k$ ). The coefficients of the double sum of formula (10) are defined by formula (11):

$$
C_{i k}=\frac{A_{k} Y_{i}\left(\frac{L}{2}\right)}{E I B_{i} \alpha_{i}^{4}\left[1-\left(\frac{\omega_{k}}{p_{i}}\right)^{2}\right]}
$$

where $A_{k}$ are the amplitude of perturbation (15), $p_{i}$ are the natural frequencies:

$$
\alpha_{i}^{4}=p_{i}^{2} \frac{\rho A}{E I}, \omega_{k}=2 \pi v_{k}
$$

and:

$$
B_{i}=\int_{0}^{L}\left(V\left(\alpha_{i} s\right)-\frac{V\left(\beta_{i} s\right)}{U\left(\beta_{i} s\right)} U\left(\alpha_{i} s\right)\right)^{2} d s
$$

where $\beta_{i}=L \alpha_{i}$, and:

$$
U(z)=\frac{\cosh z-\cos z}{2}, V(z)=\frac{\sinh z-\sin z}{2}
$$

are two of Krylov's four functions, [13]. The perturbation force function has the form:

where:

$$
F(x, t)=\sum_{k=1}^{N_{c}} A_{k} \psi_{k}(t) H\left(x-\frac{L}{2}\right)
$$

$$
\psi_{k}(t)=\sin \left(\omega_{k} t\right)
$$

Knowing the relative displacement (10), the strain and the stress in the bar is obtained:

$$
\varepsilon(x, t)=\frac{I}{W} \frac{\partial^{2} y}{\partial x^{2}}(x, t), \sigma(x, t)=\sigma(\varepsilon(x, t))
$$

To obtain the explicit expression of the stress, from (17), the relationship (5) will be used. In (17), $I$ is the area moment of inertia, and $W$ is the bar section modulus.

\subsection{Results, comments}

In this section, three results that can facilitate to the readers, an estimation of the simulation capabilities of the mathematical model defined by equations (1) - (17), but also of its deficiencies are presented.

\subsubsection{Oscillations which produce fatigue, compared to oscillations that don't produce fatigue for the bar's material}

A disturbing force of the form (4) with the intensity $F_{0}=50 \mathrm{~N}$, and the frequency $v=1 \mathrm{~Hz}$, for the oscillation which does not produce the fatigue of the bar material, and the intensity $F_{0}=65 \mathrm{~N}$, and the frequency $v=1 \mathrm{~Hz}$, for the oscillation which produces fatigue of the material is considered. The variation of the relative displacement (the deformation) in space and time is given in Figure 3, respectively Figure 4. 
Intuitively, it is expected that in the case of bending vibrations, at low amplitude and frequencies that maintain the deformation in the elastic-linear range, the maximum specific stress and deformation should have values close or even equal to those from the case of static load with the same magnitude of force. These expectations are partially confirmed. For the oscillation that does not produce the fatigue of the bar material, the magnitude of the deformation (relative displacement), as well as the strain and stress in the ends and the middle of the bar, practically coincide. By computing the maximum deflection and the stress at the middle of the bar and at the ends, for the static case, according to [14], [15] or [17], the result from the Table 1 and 2 are obtained.

Table 1 Results for the case where the bar is bent without fatigue.

\begin{tabular}{|l|r|r|}
\hline Case & \multicolumn{2}{|c|}{$F_{0}=50 \mathrm{~N}$} \\
\hline & Deflection, $\mathrm{m}$ & \multicolumn{1}{c|}{ Stress, MPa } \\
\hline Static load & 0.001091 & 152.789 \\
\hline $\begin{array}{l}\text { Vibration } \\
\text { load }\end{array}$ & 0.001091 & 154.341 \\
\hline
\end{tabular}

Table 2 Results for the case where the bar is bent with fatigue accumulation.

\begin{tabular}{|l|r|r|}
\hline Case & \multicolumn{2}{|c|}{$F_{0}=65 \mathrm{~N}$} \\
\hline & Deflection, $\mathrm{m}$ & Stress, MPa \\
\hline Static load & 0.001419 & 198.625 \\
\hline $\begin{array}{l}\text { Vibration } \\
\text { load }\end{array}$ & 0.001418 & 200.643 \\
\hline
\end{tabular}

The differences can be motivated by the fact that the magnitude of $50 \mathrm{~N}$ makes the bar work in the elastic-linear range below the fatigue limit, while the $65 \mathrm{~N}$ magnitude makes the bar work in the linear-elastic range, but in the area in which fatigue is produced.

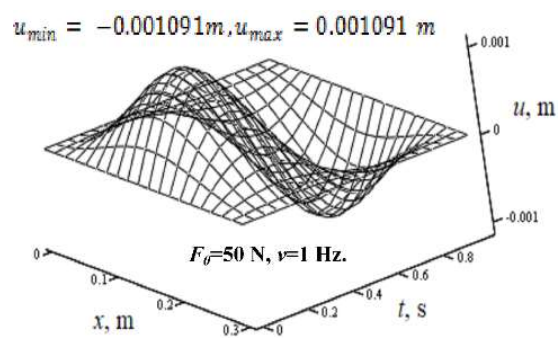

Fig. 3 Time and space dependence of the bar deformation without fatigue.

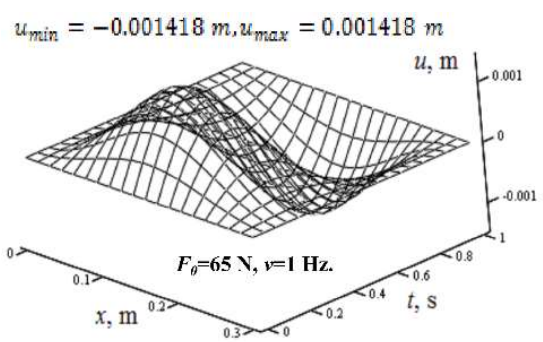

Fig. 4 Time and space dependence of the bar deformation with fatigue.

The strain and the stress space and time dependence are comparatively presented in Figure 5 and 6 , respectively 7 and 8 .

An interesting aspect to note is that fatigue has a local character both in space and in time. If the loading of the material is low and the strain and stress are located below the fatigue limit, then the ultimate strain and stress remains constant, as shown in Figures 9 and 10.

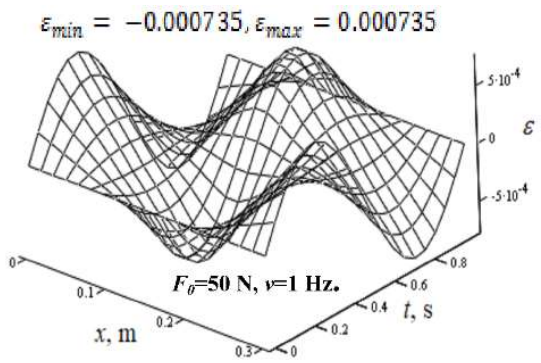

Fig. 5 Time and space dependence of the strain in the bar without fatigue.

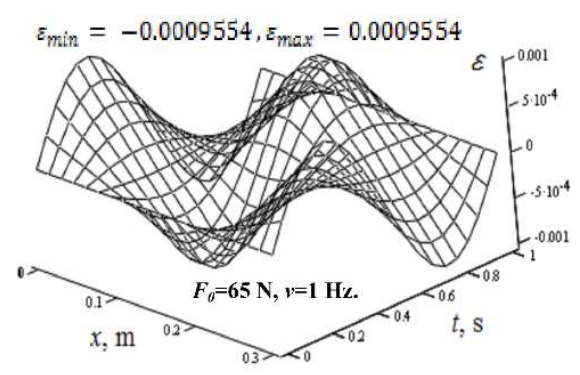

Fig. 6 Time and space dependence of the strain in the bar with fatigue. 


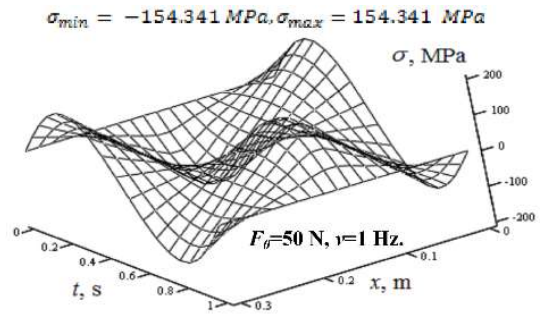

Fig. 7 Time and space dependence of the stress in the bar without fatigue.

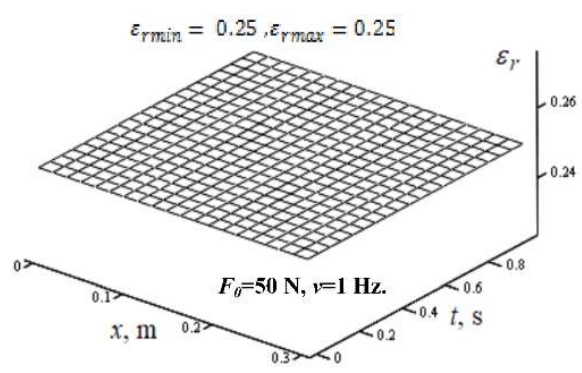

Fig. 9 Time and space dependence of the ultimate strain in the bar without fatigue.

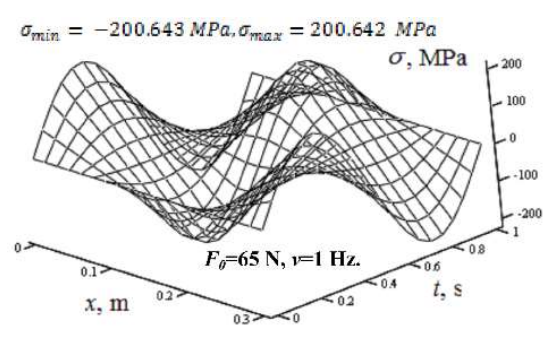

Fig. 8 Time and space dependence of the stress in the bar with fatigue.

$\varepsilon_{\text {rmin }}=0.24998797, \varepsilon_{\text {rmax }}=0.25$

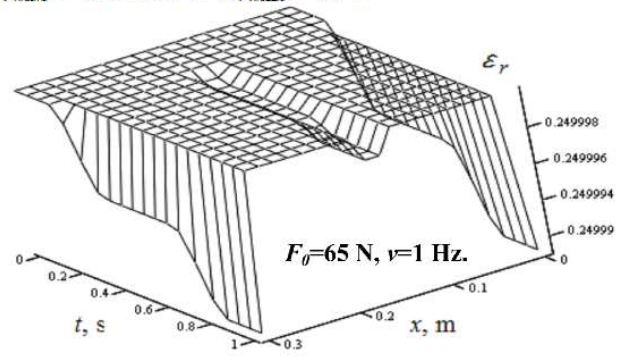

Fig. 10 Time and space dependence of the ultimate strain in the bar with fatigue.

When the load produces high stresses and deformations in the material, over the fatigue limits, in the material areas where these values are reached, the ultimate strain and stress limits decrease from initial values, as seen in Figures 10 and 12.

It can be seen that in the case of the bar examined in this article, $\varepsilon_{r}$ and $\sigma_{r}$, decreases from the initial value in the vicinity of the bar's ends and at the middle of the bar, i.e. exactly the locations where it is recommended to dimension (calculate) this type of bar.

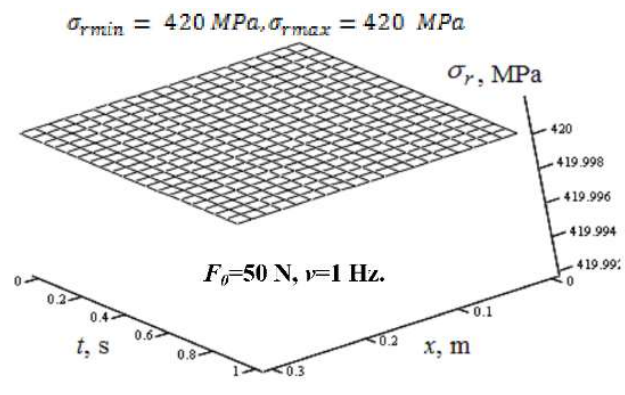

Fig. 11 Time and space dependence of the ultimate stress in the bar without fatigue.

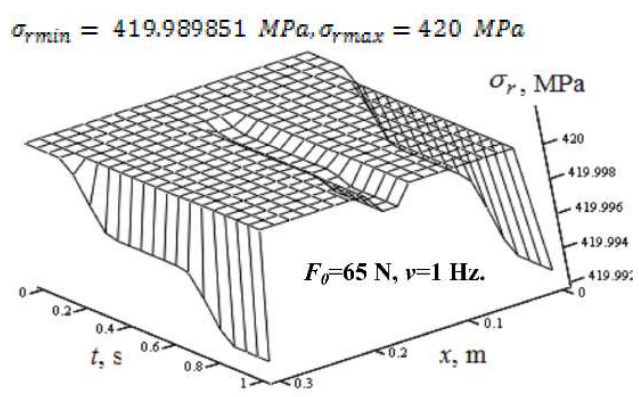

Fig. 12 Time and space dependence of the ultimate stress in the bar with fatigue. 


\subsubsection{Disturbing forces with multiple oscillating components}

In order to emphasize the behaviour of the model at the action of a disturbing force consisting of three components, of which only one that produces the fatigue of the material of the bar, the expression (15) - (16) is used with the next amplitudes: $A_{1}=5 \mathrm{~N}, A_{2}=15 \mathrm{~N}$ and $A_{3}=75$ $\mathrm{N}$, and the frequencies: $v_{1}=5 \mathrm{~Hz}, v_{2}=2.5 \mathrm{~Hz}, v_{3}=0.7 \mathrm{~Hz}$, respectively the phases: $\varphi_{1}=0$, $\varphi_{2}=0.3$, and $\varphi_{3}=0.7$.

Several quantitative and qualitative aspects of the bar response to the conjugate action of the three oscillating components of the disturbing force are shown in Figures 13-16. It is noticed that the deformation of the bar takes place in the linear elastic range, but partly in the upper part, the area in which fatigue accumulates. The deformation (strain and stress, see Figure 13 and 14) does not enter in the plastic range. The areas of the bar where fatigue accumulates are the built-in ends and the neighbourhood of the point where the disturbing force is applied (Figure 15). Accumulation of fatigue at the ends of the bar is more intense than in the middle of it, as can be seen from Figures 15 and 16.

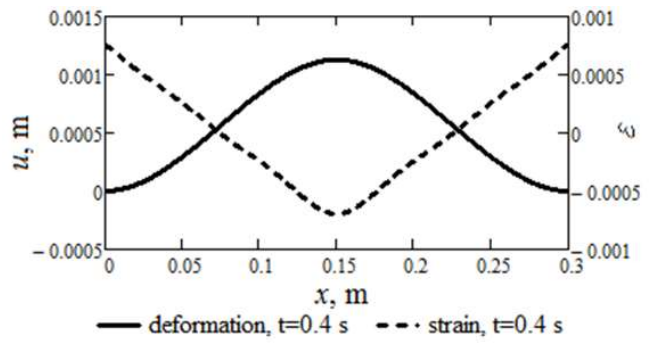

Fig. 13 Distribution of the deformation and strain along the bar.

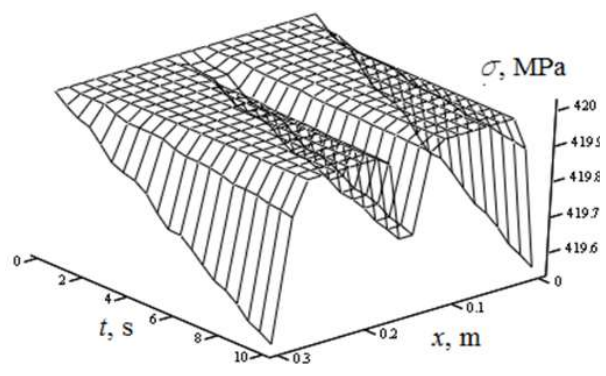

Fig. 15 Ultimate stress variation in space and time.

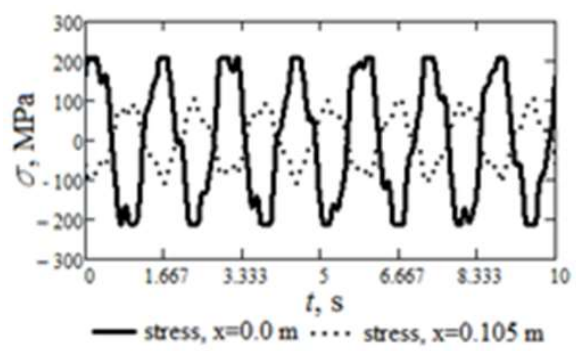

Fig. 14 Time dependence of the stress on two points on the bar.

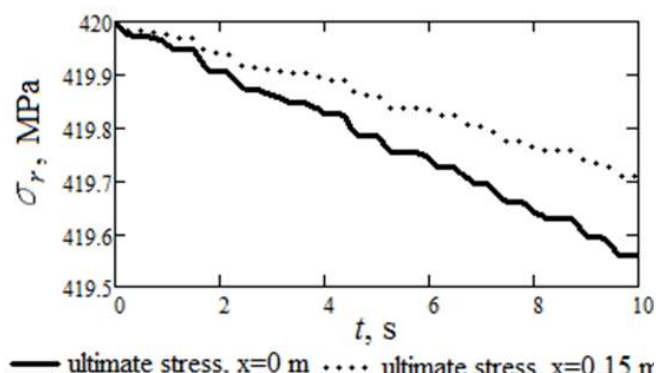

Fig. 16 Time dependence of ultimate stress in two points on the bar.

\subsubsection{Fatigue failure}

The mathematical model presented in this paper aims to model the phenomenon of fatigue for the metallic materials. As a result, this subchapter shows how to get an $S-N$ curve, traditionally called the Wöhler curve, using numerical simulation of elementary tests. The virtual testing of the endurance of the bar from Figure 1 was made by a sinusoidal disturbing force with amplitude ranging between 50 and $100 \mathrm{~N}$ and a frequency of $5 \mathrm{~Hz}$. Under these conditions, the stress in the bar, varied between $-210 \mathrm{MPa}$ and $210 \mathrm{MPa}$, being characterized by the stress ratio, $R=-1$, [11]. Although solution (10) - (16) is described by series developments, it does not require operations to solve differential equations with partial 
derivatives, the calculation, and long-term graphical representation (up to millions of cycles, $500,000 \mathrm{~s}$, for example), is still yet impossible on the computers available to the author. The main difficulty consists in solving the integral operator that appears in equations (6) and (7). In order to obtain a Wöhler curve of the material of the bar, defined by the relations (1) - (9), is assumed (hypothesis) that the failure occurs at the moment when, in the critical sections of the bar, the ultimate strength, $\sigma_{r}$, drops to the maximum value of stress, produced by the disturbing force, $F(x, t)$ in the bar. In addition, it is observed that, for times up to $10 \mathrm{~s}(50$ cycles), the ultimate stresses linearly depends on the number of cycles achieved. It is also assumed that linearity is maintained for a sufficiently large number of cycles (over 500,000). With these specifications, the Wöhler diagram shown in Figure 17 is obtained, where the medallion shows the variation in time of the disturbing force.

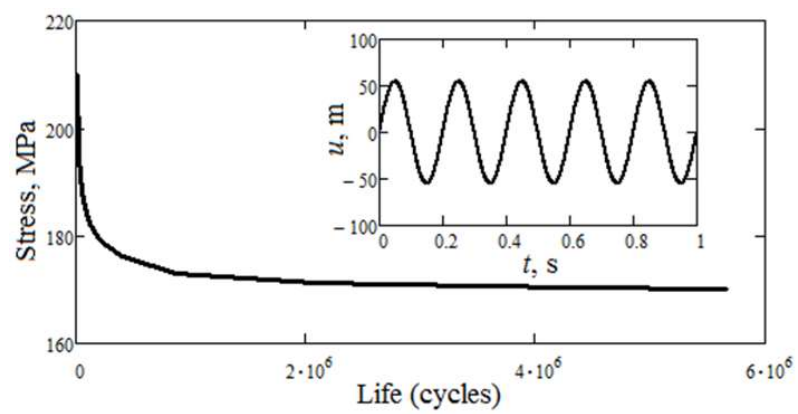

Fig. 17 Wöhler curve for the material of the bending bar, subject to sinusoidal load.

Similarly, Wöhler charts for variable loads can be obtained.

\section{Conclusions}

The mathematical model of the accumulation of fatigue in the material of the bars subject to the bending vibrations appears as a natural development of the models published by the author, [6], [7]. Accumulations of fatigue, as well as plastic deformation, are processes that irreversibly affect the internal structure of the bar. These phenomena practically describe the "leakage of life" of the bar. After every fatigue accumulation process, the bar is no longer the same as the initial one, it becomes another bar with other resistance characteristics (stress and strain breaking limits, ultimate strain and stress, $\left.\varepsilon_{r}, \sigma_{r}\right)$. The mathematical model of material fatigue presented in this paper expresses the accumulation of fatigue only by decreasing the ultimate strain and stress, $\varepsilon_{r}, \sigma_{r}$. It is possible that the fatigue phenomenon also affects the other characteristics of the material: strain and stress of flow and / or fatigue and linear elasticity, implicitly the modulus of elasticity and plasticity. Taking into account to the change in these features involves large computational complications. There is not enough experimental data to introduce such changes. Even the variation of the deformation and the ultimate tension in the material of a bar that has accumulated fatigue is quite difficult to verify.

The model defined in this article behaves satisfactorily in bending phenomena of slender bars, as long as loading makes the material work strictly in the elastic-linear range. For the future is propose to studying the bar's response at the entrance to the plastic material range. The results presented show that the accumulation of fatigue is a phenomenon unfolded over time and, generally, has local character. This means that fatigue accumulates in areas where stress and deformation take values in the upper elastic linear range, where fatigue is produced. 
The fact that the phenomenon of fatigue is a phenomenon that takes place over time does not mean that the time it is that which produces fatigue. The fatigue and implicitly the irreversible modification of the material is caused by the action of the loads, namely those loads capable of inducing in the bar, sufficiently large strain and stress. From the mechanical point of view, the material of the bar manifests its "passing of life" only to the action of these loads, which changes its structure. The value of the ultimate strain or strain can be considered, mechanically, as the internal "time" of the bar.

The mathematical model presented in this paper, although it does not require the numerical solving of complex differential equations, but only of some integral operators, has great difficulties in simulating phenomena in high time. High working times are typical for the fatigue phenomenon. In the results chapter we showed how this difficulty can be solved, at least for the time being, using a few hypotheses was showed. A Wohler diagram for the bar taken as a subject and an oscillating bending load of various magnitudes was deducted by simulation.

The model thus formulated is further tested to observe various aspects: behaviour in the plastic work range, the influence of the deformation velocity, and of the frequency of disturbing oscillations on the accumulation of fatigue, the stability of the model, etc.

This model of fatigue can be incorporated into the numerical solving programs of the deformation problems of the three-dimensional mechanical structures. The effort to introduce fatigue as a current phenomenon (whether or not fatigue is accumulating) is linked, however, to great efforts in software, but first in the experimental validation of such a model.

The main advantage of this model is that, simultaneously with solving the deformation problem of a structure, it can also provide elements that estimate its fatigue, and also localize the areas fatigue-sensitive of the structures.

Then, but not finally, the author can't stop, during the process of building this model, to reflect at the Tesla's statement, [16]: "Today's scientists have substituted mathematics for experiments, and they wander off through equation after equation, and eventually build a structure which has no relation to reality."

\section{Acknowledgement}

This work was supported by a grant of the Romanian Research and Innovation Ministry, through Programme 1 - Development of the national research-development system, subprogram 1.2 Institutional performance - Projects for financing excellence in RDI, contract no. 16PFE, and was done by "NUCLEU" Programme, developed with the support of MCI, project no. PN 19100102

\section{References}

1. A. Wohler A., Über die Festigkeitsversuche mit Eisen und Stahl, Zeitschrift für Bauwesen, Vol.20, pp. 73-106 (1870).

2. Z. Maler Z., S. Slavik, T. Marczi, M. Ruzicka, Common Mathematical Model of Fatigue Characteristics, Acta Polytechnica,Vol. 44,No. 3, pp.32-36 (2004).

3. C. Oskay, J. Fish, Multiscale Modeling of Fatigue for Ductile Materials, International Journal for Multiscale Computational Engineering, Vol. 2, No.3, (2004).

4. H. Qing, L. Mishnaevsky, Fatigue modeling of materials with complex microstructures, Computational Materials Science, Vol. 50, No. 5, pp. 1644-1650, (2011).

5. N. Stojkovic, R. Folic, H. Pasternak, , Mathematical model for the prediction of strength degradation of composites subjected to constant amplitude fatigue, International Journal of Fatigue, Vol. 103,pp. 478-487 (2017). 
6. P. Cardei, Material with Integral Memory, Revue Roumaine des Sciences Techniques, serie de Mecanique Appliquee, Vol. 52, No. 5 (1993).

7. P. Cardei, "Models for bodies with unsteady quality," Revue Roumaine des Sciences Techniques Serie de Mecanique Appliquee, Vol. 45, pp. 97-109, (2000).

8. H. F. Brinson, L.C. Brinson, Polymer Engineering Science and Viscoelasticity An Introduction, New York: Springer, (2015).

9. R. M. Jones, Deformation Theory of Plasticity, Blacksburg, Virginia: Bull Ridge Publishing, (2009).

10. Gh. Buzdugan, Rezistenta Materialelor, Bucuresti: Editura Tehnica, (1980).

11. F.C. Campbell, Elements of Metallurgy and Engineering Alloys, ASM International, (2008).

12. S. Timoshenko, Vibrations Problems in Engineering, New York: D. Van Nostrand Company, Inc., (1937).

13. Gh. Buzdugan, L. Fetcu, M. Rades, Vibratii Mecanice, Bucuresti: Editura Didactica si Pedagogica, (1982).

14. Hutte, Die Grundlagen der Ingenieurwissenschaften, Berlin: Springer-Verlag, (1989).

15. V. Drobota, Rezistenta Materialelor, Bucuresti: Editura Didactica si Pedagogica (1982).

16. N. Tesla, Modern Mechanics and Inventions (1934).

17. S. Timoshenko, Strehgth of Materials, New York, Toronto, London: Lancaster Press Inc. (1948). 